\title{
Medical Decision Support Systems and Semantic Technologies in Healthcare
}

\author{
Kukhtevich I.I.* \\ Penza State Institute of Advanced Medical Education \\ Penza, Russia \\ gvv17@ya.ru
}

\author{
Goryunova V.V. \\ Penza State University \\ Penza, Russia \\ gvv3317@ya.ru
}

\begin{abstract}
The support system for making medical decisions is a dynamically developing knowledge base at the intersection of medical sciences, medical institutions, doctors, etc. The knowledge base should be replenished, as clinical cases, recommendations, and treatment algorithms are being improved. In addition, in the current situation there is a clear trend towards personalized medicine, which will be the most productive. The article discusses the aspects of using medical decision support systems in the practice of medical organizations. The necessity of using the Unified State Health Information System is emphasized. The aspects of standardization of medical information systems and the use of ontologies, which are open standards for the description of the semantic components of the treatment process, are noted. LOD technology specifications are given. The possibility of combining distributed semantic repositories created by various organizations (health authorities, medical organizations, research centers, etc.) into a single network is noted. The possibility of using UMLS for the development of information retrieval systems, for registration of patients, to facilitate communication between various systems that analyze biomedical information is being considered. The task of the near future is to develop a medical information system as an intellectual physician assistant, including the introduction of clinical guidelines, automatic quality control of medical care and other areas that can significantly improve the quality of medical care and reduce the number of medical errors. Three groups of criteria for the quality of the doctor's activity are defined: semantic, temporary and effective. It is noted that the widespread use and constant replenishment of modern clinical knowledge is based primarily on clinical recommendations. The experience of using the register for stroke is presented and the results of clinical recommendations tested in practical public health in the field of neurology are taken into account.
\end{abstract}

Keywords - digital economy, healthcare, medical decision support systems, semantic technologies, neurology

\section{INTRODUCTION}

When considering modern organization management paradigms [1], the transition from an economy using information technologies to a digital economy is actively

\author{
Goryunova T.I. \\ V.A. Trapeznikov Institute of Control Sciences of Russian \\ Academy of Sciences \\ Moscow, Russia \\ tatjanagoryunova20@gmail.com
}

\author{
Zhilyaev P.S. \\ Penza State University \\ Penza, Russia \\ itmmbs17@mail.ru
}

discussed [2]. In the first case, the task of informatisation of existing and planned business processes is solved. In the second, an organization is being constructed in a high-tech and ultra-fast information environment that ensures the creation of targeted products for consumers and added value for manufacturers.

The main thing here is the emergence of a special resource - Big Data and the possibility of their analysis and construction based on semantic technologies and end-to-end business processes. Big data is practically unlimited, coming from different sources, describing dissimilar objects in various sign and ontological systems, appearing and existing in real time [3]. For example, data on the dynamics of requests for specific medicines in online pharmacies can be used to predict seasonal diseases and, accordingly, plan stocks of medicines.

Thus, the traditional approach to the design of medical information systems based on the study of an organization is replaced by the construction of a new business model for this organization that effectively uses IT services [4].

Big data in medicine is associated with medical decisionmaking and scientific research.

Effective medical decision support systems (MDSS) are a dynamically developing knowledge base at the intersection of biomedical science, medical institutions, doctors, etc. [5]. It is clear that care must be taken to select the right drugs, because not everyone will be equally effective for a particular person with a unique genetic code and characteristics of the body. The knowledge base should be updated, because clinical cases, recommendations and treatment algorithms are being improved. Plus, there is a clear tendency towards personalized medicine, which will develop using intelligent information technologies. In this case, the knowledge base with MDSS is not a set of rules, but a specialized software product that uses semantic and cloud technologies.

The article presents practical examples of using the stroke register, which takes into account the results of 
clinical recommendations tested in practical healthcare. Automatic formation of the register using interface forms greatly facilitates the introduction of the register into the clinical practice of neurologists. The stroke register minimizes the time spent, because it contains the scenarios necessary for a doctor to make a decision, as well as a list of calculators and scales for determining the severity of the condition, the severity of functional and neurological deficits, and calculating the risk of bleeding when prescribing anticoagulant therapy.

\section{ANALYSIS OF MODERN METHODS OF IMPLEMENTING SEMANTIC TECHNOLOGIES IN HEALTH CARE}

As in other countries, in the field of healthcare in Russia, new information technologies are being developed to support the processes of diagnosis and treatment and the accumulation of new knowledge on the use of drugs. The need to quickly obtain quality information to address both medical and managerial issues is constantly growing. There is a rapidly developing contradiction between the growing demand for information, its volume and the impossibility of its effective use.

To solve such problems, an international standard for publishing data on the Web, the Linked Open Data (LOD) standard, created and actively developed as the technology of the semantic web, has been developed and is actively used.

Any data is converted to the LOD format - structured (databases, tables) and unstructured (web pages, documents of various formats).

The ease of integrating data from different sources allows connecting to different data producers. Linking data from various sources increases the value of the information provided [6].
In this format, overseas (in the UK, USA, Canada, Germany, Sweden, Hungary, etc.) a powerful network of linked databases has been created for various fields of knowledge that are created and used by both state and commercial structures.

One of the most important advantages of LOD technology is the possibility of combining into a common network of distributed semantic storages created by various organizations (public health authorities, medical organizations, research centers, professional communities, universities, etc.) based on common open standards. As international experience shows, this allows the system to self-develop, constantly replenishing it with data and improving their quality.

The Unified Medical Language System (UMLS) is used as a collection of many controlled dictionaries in the field of biomedicine. UMLS is also used in other areas for natural language processing. The initiative to implement the project belongs to Donald Lindbergh. The UMLS development project is currently supported by the US National Library of Medicine, updated quarterly, and can be used for free.

UMLS can be used to develop information retrieval or patient registration systems, to facilitate communication between different systems, or to develop systems that analyze biomedical literature. For many of these applications, UMLS must be used in user-defined form, for example, by excluding certain source dictionaries that are not relevant to the application.

Some examples of controlled dictionaries included are ICD-9-CM, ICD-10, mesh, SNOMED CT, LOINC, WHO adverse drug reaction terminology, UK Clinical Terms, RxNORM, Genes Ontology, and OMIM). The approximate composition of UMLS is presented in Figure 1.

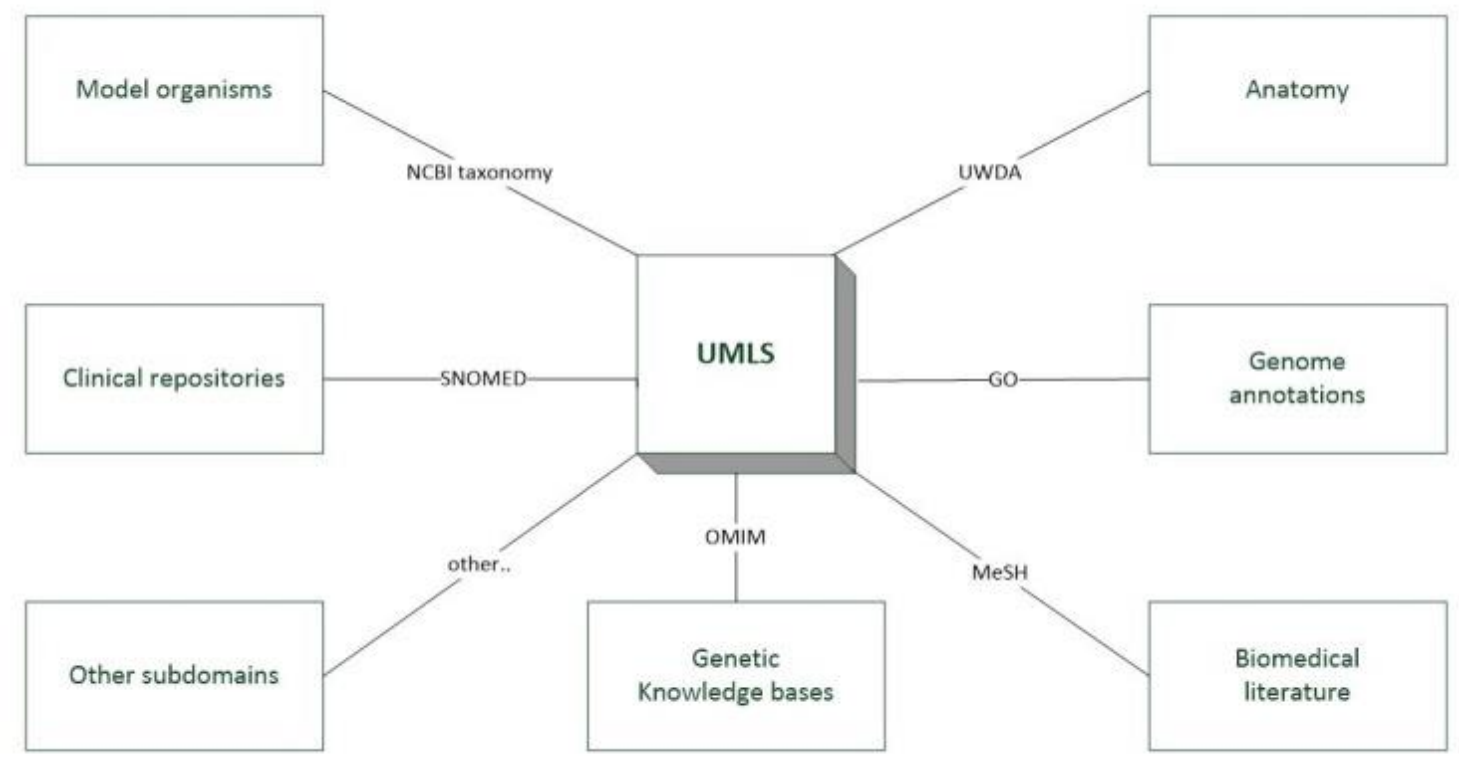

Fig. 1. UMLS and controlled dictionaries 
In modern implementations, UMLS includes the following components:

- "metathesaurus", is a collection of concepts and terms from various controlled dictionaries, and their relationships;

- "semantic networks", is a set of categories and relationships that can be used to classify entries for Metathesaurus;

- "vocabulary specialist", a tool based on lexicographic information for use in natural language processing;

- some of the supporting software.

Metathesaurus is based on concepts. Each concept has certain attributes that determine its meaning and is associated with the corresponding concept names in different source dictionaries. Relationships between concepts are presented, for example hierarchical, such as "is a" for subclasses and "is part of" for subunits, and associative, such as "is caused by", etc. [7]. All hierarchical information from the source dictionaries is stored in Metathesaurus. Metathesaurus concepts can also refer to resources outside the database.

Metathesaurus is created by automated processing of computer versions of source dictionaries, followed by editing by an expert.

The semantic network is a catalog of semantic types and semantic relationships. This is a large classification, which contains almost 140 skmantic types and more than 60 relationships.

At least one semantic type (category) is assigned to each concept in Metathesaurus. Semantic relations are defined between members of different semantic type.

The main semantic types are organisms, anatomical structures, biological functions, chemicals, events, physical objects and concepts or scenes. The relationships between semantic types provide the network structure [8]. The main relationships between semantic types are "is-a" relationships. These relations establish a hierarchy of types and make it possible to find the most semantic type that can be assigned to this concept of a metaosaurus [9]. The network also has 5 main categories of non-hierarchical (or "associative") relationships:

- "physically connected";

- "spatially connected";

- "temporarily connected";

- "functionally related";

- "conceptually related."

Information about the semantic type includes the following components: identifier, definition, examples, hierarchical information about the covering semantic types and their associative relationships. Associative relations record the fact that the corresponding relational statement makes sense (although it does not have to be true in all cases).
The lexicon contains information about vocabulary (English), biomedical terminals, terminals included in MEDLINE in UMLS and Metathesaurus. Each entry contains: syntactic (how words create meaning and add together), morphological (form and structure) and spelling (spelling) information. Using Java programs, the lexicon works with variations in biomedical texts, which can be useful when searching the Internet and electronic medical records.

The specialized dictionary is available in two formats: the single record format and the relational table format. The "single record" format contains "slots" and "placeholders". "Slot" is an element, and "placeholders" are the values associated with this slot for this entry. The format of the "relational table" is poorly normalized and contains a large amount of duplicate data.

In addition, in modern UMLS implementations, auxiliary software tools are included:

"MetamorphoSys" is a program that can be used to customize Metathesaurus for specific applications, for example, by excluding certain source dictionaries.

"lvg" is a program that uses specialized vocabulary to generate lexical variants of a given term and to support parsing of text in natural language.

"MetaMap" is an online tool that, when defining an arbitrary piece of text finds and returns the corresponding Metathesaurus concepts.

"Metamap Transfer" (MMTx) provides the same functionality as a Java program.

"Knowledge Source Server" is an interactive application that allows viewing Metathesaurus.

\section{III.METHODOLOGY FOR USING MEDICAL DECISION SUPPORT SYSTEMS}

During the treatment and diagnostic process, the doctor pursues certain goals, takes active diagnostic and treatment actions that affect the patient's condition. Active actions aimed at achieving the goal are usually called management [10].

The doctor at each step of the diagnostic process selects a subset of the elements (diagnostic tests, laboratory tests, prescribing drugs). The choice is based on the knowledge accumulated by medicine about how to treat this disease, based on the physician's own experience.

In Russia, with the creation and development of the Unified State Health Information System (USHIS), information technologies have become widely used in the practice of medical organizations (MO). A certain standardization of medical information systems (MIS) is observed: instead of hundreds of different, often highly specialized and diverse developments, standard products come.

The digital process forms certain attributes that arise at different stages - from the establishment of an electronic medical record with personal data, prescription of 
medications, diagnosis and analysis. All these data are aggregated, stored, then depersonalized [11] and stored in the regional MIS and USHIS. There are a lot of these processes, they are different - there are paid services, there are free ones due to compulsory health insurance $(\mathrm{CHI})$, etc.

The main task of MIS at the current stage is the transition to the preferential management of electronic medical records of patients, the accumulation of primary medical information in electronic form and the automation of basic tasks.

Among the basic tasks[12] such aspects as reducing the non-core costs of medical personnel for maintaining documentation, the automatic generation of medical statistics, the delivery of registers for $\mathrm{CHI}$, the automation of drug accounting, etc can be attributed.

The task of the near future is the development of MIS into an intellectual physician assistant, including the introduction of clinical guidelines, automatic quality control of medical care and other areas that can significantly improve the quality of medical care and reduce the number of medical errors. The medical decision support system, like adding or improving MIS, is a very important tool for reforming and improving the work of the healthcare industry.

MIS provides 3 levels of development: minimal, basic and advanced. The minimum level is those functions, without which it is impossible to call this software product a complete medical information system. The basic level is a list of functions sufficient to implement a sound MIS and providing the doctor and nurse with the necessary functions for its management; replacing paper and pen with an electronic way of maintaining medical records.

An advanced level is the maximum list of what an MIS developer should provide for medical organizations, including support for making medical decisions. Currently, a sufficiently detailed description of the functional capabilities in various areas of the MO is presented, but there is no description of specific functions that could be attributed to supporting the adoption of medical decisions.

In general, the term "Decision Support System (DSS)" refers to a computer system that, through the collection and analysis of information, can influence decision-making processes in various fields of human activity [13]. In health care, such decisions are referred to as the amended term "medical decision support systems" (MDSS).

Intellectual support for the adoption of medical decisions has been used for a long time, and the early, traditionally applied forms include maintaining electronic medical records.

Simplification and acceleration of the formation of medical documents is the minimum level of development of MIS, which allows talking about at least some help to the doctor from the information system. Strictly speaking, conducting MIS is not a support system for making medical decisions, but without MIS it is not possible to talk about any kind of informatisation of medical work.

To the minimum necessary functions of conducting EMC, we include the automated formation of a medical document based on the established clinical diagnosis, formatlogical control while maintaining medical documents for completeness and correctness of their filling.

For example, the MIS should check and prevent errors such as: an incorrectly established ICD-10 diagnosis according to gender and age, incorrect filling in the fields of a medical document (weight, height, examination statistics, dates beyond acceptable limits, etc.), failure to comply with the clinical minimum during a medical examination (for example, during the initial examination, the doctor did not collect anamnesis or did not indicate the condition and severity of the patient, etc.).

In fact, all these functions do not really help the doctor make any decision. They rather insure him against a "routine" error in the preparation of the medical document. At the same time, it should be noted that a detailed and informatively drawn up medical record is an important contribution of the MIS to the continuity of medical care and, as a consequence, to its quality.

Quality criteria are those reference points by which a doctor's work test can be performed. They are divided into three groups:

- semantic, substantial (characterize the actions of a specialist);

- time (time intervals in which the doctor must meet);

- effective (achievement or failure to achieve a result, the appearance or absence of complications in a patient, etc.).

Quality criteria are formal indicators that should be used during inspections by regulatory authorities and medical insurance organizations.

For each completed case of treatment, an integral quality score should be set, derived taking into account all three of these groups of criteria. On average, about 15 quality criteria are developed for each disease or group of diseases.

The automation of medical appointments is perhaps the most common form of support for medical decision-making in IIAs at the moment. Most often, this functionality is presented in the form of the so-called "electronic assignment sheets" (EAS).

The main task that is solved by the introduction of EAS is the implementation of various functions that help prevent erroneous actions or inaction in relation to the life and health of patients

Two main applied approaches to the construction of decision support systems can be distinguished: decision making on precedents and on models.

In the model approach, as in any generalization, some features of the behavior of the system at each specific point in the solution space are lost.

When relying on a precedent, as experience shows, it is possible to take into account these local features, which often makes it possible to obtain more accurate solutions 
It is a case-based approach that will allow creating effective and accurate systems for supporting the adoption of medical decisions in the near future.

The published results indicate the actual clinical effect of such functions, which is to reduce the risks of medical errors and increase the efficiency of the doctor.

In order for the MIS database to support the automation of medical appointments, the following directories should be used in it:

- ICD-10.

- Directory of medical services (a list of diagnostic, therapeutic and advisory activities that may be provided in the Moscow Region, this should not be confused with a classifier of services approved at the federal level by order of $1664 n$ ).

- Directory of medical standards.

- Directory of drugs based on INN.

Two main approaches to the use of ontologies in MIS have been studied [14]. The first of these involves the use of ontology at the design and development stage of MIS. In the second approach, the ontology governs all aspects and components of the MIS already at the stage of its functioning. In the first case, they talk about the ontologydriven development of MIS, in the second - about the ontology-driven information system.

In this approach, the MIS consists of three types of components: an application program, a data warehouse (database and / or knowledge base) and a user interface. These components, connected together, provide the required system functionality. There are two orthogonal aspects of the influence of ontology on MIS: the temporal (temporal) aspect, showing at what stage of the MIS life cycle the ontology is used - at the design stage or at the stage of system operation, and the structural (constructive) aspect, reflecting the way the ontology affects the main components of MIS.

\section{IV.QUESTIONS USE OF MEDICAL DECISION SUPPORT SYSTEMS AND STROKE REGISTER IN NEUROLOGY}

Vascular diseases of the brain are the most important medical and social problem of modern society, due to their prevalence, high mortality, significant indicators of temporary labor losses and primary disability.

In economically developed countries (USA, UK, Scotland and the Netherlands), medical care for stroke accounts for about 3-4\% of all health care costs.

In Russia, the cost of treating 1 patient who has had a stroke, including inpatient treatment, medical and social rehabilitation and secondary prevention, is over 160 thousand rubles in year. One of the ways to improve the quality and safety of medical care is the development of MDSS.

The widespread use and constant replenishment of modern clinical knowledge in MDSS is based primarily on clinical guidelines. In a systematic review, it was found that in $94 \%$ of cases, these systems significantly improve the quality of clinical practice.

The authors presented studies on the implementation of the MDSS Clinical Guidance and Audit Automation System (SACRAL), based on clinical guidelines, for the management of patients with acute cerebrovascular accident at the inpatient and outpatient stages.

SACRAL includes an algorithm for differential diagnosis of stroke and protocols for making a personalized decision on the management of patients with different types of stroke in all stages of inpatient care, monitoring the implementation of medical interventions, evaluating early and long-term results of treatment. In addition, the automated system allows generating analytical and statistical reports, as well as generating quality registers in automatic mode.

MDSS includes an algorithm for the differential diagnosis of acute cerebrovascular accident and protocols for making a personalized decision on the management of patients with different types at all stages of inpatient care, monitoring the implementation of medical interventions, evaluating early and long-term treatment results. In addition, the automated system allows generating analytical and statistical reports, as well as generate quality registers in automatic mode.

The SACRAL program is based on formalization, subsequent coding of clinical recommendations according to the "if ... then ..." rule and the formation of decision tables based on an automated analysis of the entered data. The demographic and clinical data of the patient, the results of diagnostic tests are presented in the form of questions and answer options. A set of questions and answers forms a form filled out by users (attending physicians), on the basis of which protocols and recommendations are formed. The idea of introducing clinical recommendations is to give the doctor a unified, verified at the federal level set of specific reliable practical recommendations on patient management tactics and thereby facilitate the work of the doctor and improve its quality.

Scenarios contain all the questions necessary for making a decision, as well as a list of calculators and scales for determining the severity of a condition, the severity of functional and neurological deficits (Glasgow coma scale, Rankin scale, NIHSS), calculation of bleeding risk when prescribing anticoagulant therapy.

Filling out the scenario in the first 24 and 72 hours of ischemic stroke (IS) based on the patient's risk factors, objective instrumental and laboratory examination data, determining the pathogenetic subtype of the stroke, decisionmaking is supported by the timing and extent of the followup examination, treatment measures and early secondary prevention of stroke

A protocol with recommendations is automatically generated in the system.

An important advantage of the system is the presence of an epicrisis configurator in it, which is a branched tree of 
questions and answers. Specific scenarios may include an arbitrary subset of questions and answers to them that can be edited by medical experts.

The database of the system is a source of information for analyzing the quality of patient management and the automatic formation of a register with the inclusion of all entered patient data. According to the register, an expert doctor can generate a report for any period of time. When developing a register for stroke, the results of clinical registers tested in practical healthcare were taken into account. The automatic formation of the register as the forms of the program are completed significantly simplifies the implementation of the register in clinical practice, minimizing the time spent and providing more accurate and complete filling in (since the data is entered by the attending physician), and saves the financial resources of the hospital [15].

\section{CONCLUSION}

The use of intellectual decision support and a knowledge base can be expanded using various mechanisms, primarily symbolic web applications (web services, data exchange, etc.). This approach is also of particular importance for integration with external systems, for example, a system for assessing "environmental health" [16].

The medical decision support system, as an addition to or improvement of MIS, is a very important tool for reforming and improving the work of the healthcare industry.

The development of MDSS corresponds to the general strategy of innovative development of Russia [17].

The knowledge base of the system is a source of information for analyzing the quality of patient management and automatic reporting. When developing the register for stroke, the results of clinical recommendations tested in practical public health were taken into account. In the integration model, the rule base works with a semantic network, the composition of the concepts and relations of which is determined by the ontology.

In particular, the automatic formation of the stroke register as the program forms are filled out significantly simplifies the work of the doctor and introduction into clinical practice. Filling out the scenarios based on the patient's risk factors, objective instrumental and laboratory examination data, determination of the pathogenetic subtype provides decision support on the timing and extent of the follow-up examination, treatment measures and early secondary prevention of stroke.

\section{References}

[1] V. Burkov, M. Goubko, N. Korgin, D. Novikov, Introduction to Theory of Control in Organizations. New York: CRC Press, 2015, $352 \mathrm{p}$.

[2] Program "Digital Economy of the Russian Federation". Retrieved from:

http://static.government.ru/media/files/9gFM4FHj4PsB79I5v7yLVuP gu4bvR7 M0.pdf (accessed: 02/14/2020).

[3] V.V. Goryunova, O.V. Lukinova, T.I. Goryunova, "Ontologically oriented methods for integrating modular systems ERP", Pacific Conf. on computer technologies and applications. Vladivostok, 2017, pp. 208-210.

[4] D. Novikov, A. Chkhartishvili, Reflexion and Control: Mathematical Models. London: CRC Press, 2014, 298 p.

[5] T. Gavrilova, I. Leshcheva, Design of ontologies and individual cognitive features: a pilot study, Expert systems with applications, available online information. DOI: $10.1016 /$ j.eswa.2015.01.008

[6] V.A. Fokin, T.V. Novikova, Y.S. Pekker, V.V. Novitsky, The concept of a database of scientific research in medicine, Siberian Med. J., vol. 15 , no. 4, pp. 32-34, 2000. Tomsk.

[7] D.L. Brock, E.V. Schuster, S.J. Allen, P. Kar, "Introduction to semantic modeling for logistics systems", J. of Busin. Logist., vol. 26, no. $2,2009$.

[8] V. Omachonu, N., "Innovation in Healthcare Delivery Systems: A Conceptual Framework", The Public Sector Innovat. J., Vol. 15, no. 1, Article 2, 2010

[9] "World Health Organization. Telemedicine: opportunities and developments in Member States", Report on the second global survey on eHealth. 2009.

[10] D. Novikov, Management Methodology. New York: Nova Sci. Publ., 2013, p. 76

[11] New frontiers in the modeling and analysis of information and production systems - incentive mechanisms, competency management, knowledge-based production, Series Reference library of intelligent systems, ed. P. Rozewski, D. Novikov, O. Zaikin, N. Bakhtadze. Berlin: Springer, 2016, 268 p.

[12] E. Brignolfsson, B. Kahin, Understanding the digital economy: data, tools and research, Cambridge: MIT Press, 2000, pp. 1-10.

[13] G.B. Kleiner, "Systemic paradigm and systems management", Rus. Office J., vol. 3, no. 6, pp. 27-50, 2001.

[14] V.V. Goryunova, "Modular ontological system technology in intelligent information systems", Information-measuring and operating systems, vol. 10, pp. 56-61, 2010.

[15] D.A. Shageev, T.A. Chukhontseva, "Universal express methodology for assessing and making managerial decisions of situational problems at the enterprise", Bull. of the Voronezh State Univer. of Engineer. Technol., vol. 81, no. 2, pp. 359-376, 2019. Retrieved from: https://doi.org/10.20914/2310-1202-2019-2-359-376

[16] G.S. Merzlikina, "The economic effectiveness of the "ecological health" of the region", Bull. of the Voronezh State Univer. of Engineer. Technol., vol. 81, no. 2, pp. 312-319, 2019. https://doi.org/10.20914/2310-1202-2019-2-312-319

[17] Yu.V. Zhuravlev, I.V. Kuksova, E.A. Hubertov, L.I. Churikov, "Assessment of the innovative development of the Russian Federation based on indicators of the concept and strategy of 2020", Bull. of the Voronezh State Univers. of Engineer. Technol., vol. 81, no. 2, pp. 377-382, 2019. Retrieved from: https://doi.org/10.20914/23101202-2019-2-377-382 